\title{
Erratum to: NY-ESO-1 autoantibody as a tumor-specific biomarker for esophageal cancer: screening in 1969 patients with various cancers
}

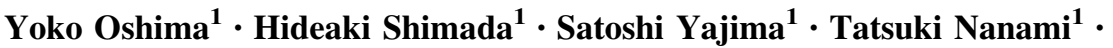 \\ Kazuyuki Matsushita ${ }^{2} \cdot$ Fumio Nomura $^{2}$ - Osamu Kainuma ${ }^{3}$ Nobuhiro Takiguchi ${ }^{3}$. \\ Hiroaki Soda ${ }^{3}$ - Takeshi Ueda ${ }^{4}$ - Toshihiko Iizasa $^{5}$ - Naoto Yamamoto ${ }^{6}$. \\ Hiroshi Yamamoto ${ }^{3}$. Matsuo Nagata $^{3} \cdot$ Sana Yokoi $^{7} \cdot$ Masatoshi Tagawa $^{8}$. \\ Seiko Ohtsuka ${ }^{1}$ - Akiko Kuwajima9 ${ }^{9}$ Akihiro Murakami' ${ }^{9}$ Hironori Kaneko ${ }^{1}$ \\ Published online: 16 May 2015 \\ (C) Springer Japan 2015

\section{Erratum to: J Gastroenterol} \\ DOI 10.1007/s00535-015-1078-8
}

The conflict of interest statement should read as follows:

Conflict of interest Akiko Kuwajima and Akihiro Murakami are employees of Medical \& Biological Laboratories Co., Ltd., Nagoya, Japan. The other authors declare that they have no conflict of interest.

The online version of the original article can be found under doi:10.1007/s00535-015-1078-8.

Hideaki Shimada

hideaki.shimada@med.toho-u.ac.jp

1 Department of Surgery, School of Medicine, Toho University, Tokyo, Japan

2 Molecular Diagnosis, Graduate School of Medicine, Chiba University, Chiba, Japan

3 Division of Gastroenterological Surgery, Chiba Cancer Center, Chiba, Japan

4 Division of Urology, Chiba Cancer Center, Chiba, Japan

5 Division of Thoracic Disease, Chiba Cancer Center, Chiba, Japan

6 Division of Breast Surgery, Chiba Cancer Center, Chiba, Japan

7 Division of Clinical Genomics, Chiba Cancer Center, Chiba, Japan

8 Division of Pathology and Cell Therapy, Chiba Cancer Center, Chiba, Japan

9 Medical \& Biological Laboratories Co., Ltd, Nagoya, Japan 